\title{
Molecular Characterization of Elastic Deformation in Elastin Mimetic Peptide [LGGVG] $]_{3}$
}

\author{
Cheng SUN* \\ College of Physical Science and Technology, Dalian University, Dalian, 116622, China \\ crossref http://dx.doi.org/10.5755/j01.ms.24.3.18432
}

Received 22 June 2017; accepted 30 September 2017

\begin{abstract}
Owing to the dramatic elasticity of elastin mimetic peptides, attention is accumulating to the molecular properties of the elastic polymers. This work reports on the molecular characterization of elastic deformation in an elastin mimetic peptide $[\mathrm{LGGVG}]_{3}$ via molecular dynamics simulation. The elastic deformation process is captured by pulling, holding and releasing the peptide. The molecular properties of the released peptide from being pulled are observed almost identical to that of the relaxed peptide, and this indicates the excellent elasticity of the elastic biopolymer. From the results, it is suggested that the scheme implemented in this work could be used in studies of other elastic polymers. Keywords: elastin mimetic peptide, $[\mathrm{LGGVG}]_{3}$, molecular dynamics simulation.
\end{abstract}

\section{INTRODUCTION}

Elastin mimetic peptides are stimulus responsive peptide polymers that resemble the thermal and mechanical properties of elastin - an extracellular insoluble protein that is responsible for the remarkable elasticity of many vertebrate tissues [1-5]. Thanks to the temperature sensitive behavior and dramatic elasticity, the peptides have gained much attention in many research areas for various stimulus responsive applications, including drug delivery, polymer design, tissue engineering, etc. For example, elastin like polypeptide scaffolds for local delivery of antibiotics were prepared and characterized [6], a temperature dependent elastin-like peptide modified dendrimer was synthesized for drug delivery [7], the elastin like polypeptide polymers containing tumor targeting AP1 peptide was constructed [8], novel elastin like polypeptide-collagen composites were prepared and characterized [9], and sequence length dependent surface and adsorption characteristics of elastin like polypeptide coatings were investigated for developing blood contacting biomaterials [10]. So far, most applications have been focused on the temperature-induced behaviors of the peptides; for instance, a quantitative model that predicted the transition temperatures of elastin like peptides [VPGXG] was proposed [11], and the transition temperatures of elastin like polypeptide fusion proteins were also predicted [12]. While studies on the polymers' stress related properties are few. It is therefore significant to probe the molecular structures and dynamics during the deformation of the peptide upon stain, and this is the scope of this work.

To date, several commonly studied elastin mimetic peptides have included repeating motifs of VPGVG and LGGVG, and this is mainly because that the tandem repeats have been found in the hydrophobic domains of elastin and the regions have been believed to bear the

\footnotetext{
* Corresponding author. Tel.: +86-411-87402712

E-mail address: suncheng@dlu.edu.cn (C. Sun)
}

source of elasticity [13]. For example, the peptides $[\mathrm{VPGVG}]_{n}$ was shown to exhibit the same temperature induced structural transitions as elastin by circular dichroism (CD) [14], and a thermal hysteresis in the backbone and side-chain dynamics of the peptide $[\text { VPGVG] }]_{3}$ was also revealed by ${ }^{2} \mathrm{H}$ nuclear magnetic resonance (NMR) [15]. Besides, the structure of the peptide poly[LGGVG] was characterized by CD and NMR [16], and the peptide [LGGVG] $]_{n}$ was also found to have structural similarities to elastin by NMR $[17,18]$.

Among the techniques that characterize the properties of elastin mimetic peptides, molecular dynamics (MD) simulation has been proven to be a powerful, convenient scheme in determining the molecular structural and dynamical properties of the peptides. For instance, MD simulations were performed on the elastin like peptide $[\text { VPGVG] }]_{18}$ to study the dominant source of elastin's elasticity and the molecular basis for the inverse temperature transition of elastin $[19,20]$. MD simulations were also employed on the elastin mimetic peptide $[\mathrm{LGGVG}]_{3,7}$ to study the inverse temperature transition and the elastomeric force development of elastin [21]. In this work, MD simulations were performed on the elastin mimetic peptide $[\mathrm{LGGVG}]_{3}$ to characterize the elastic deformation of the peptide.

\section{METHODS}

The MD simulations were performed using the GROMACS package [22]. The peptide was terminated by the amine $\left(-\mathrm{NH}_{2}\right)$ group at the $\mathrm{N}$-terminus and the carboxyl $(-\mathrm{COOH})$ group at the C-terminus. For all simulations, the peptide was solvated with water molecules in a box extending at least $1 \mathrm{~nm}$ in all directions, and the temperature of the system was regulated at $37{ }^{\circ} \mathrm{C}$ using a Berendsen thermostat [23]. The OPLS-AA/L force field model [24] was used and the SPC216 water model [25] was employed in this work. Initial atomic velocities were generated from a Maxwellian distribution and periodic boundary conditions were implemented. For all 
simulations, an energy minimization was first performed using the steepest descent method to remove overlapping atoms [26], and short simulations restraining the peptide position and equilibrating the temperature and pressure were then carried out before executing the production simulations.

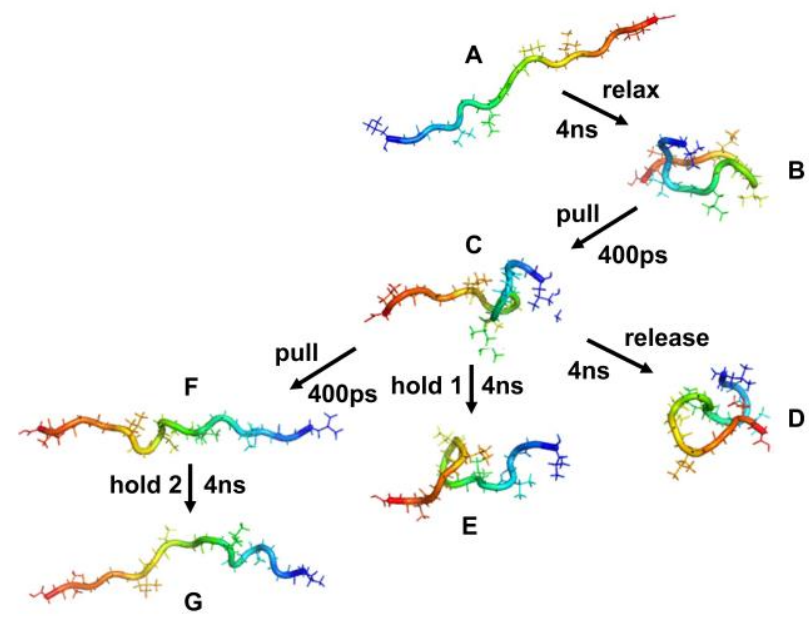

Fig. 1. Schematic flowchart of the simulations performed on the peptide $[\mathrm{LGGVG}]_{3}$ in this work. The time evolution of the simulations is indicated by arrows. The peptide states labelled A, B, C, D, E, F and G are the final structures determined from each simulation, detailed in the text

In this work, several constrained and unconstrained simulations were performed to mimic the pulling and releasing process of this elastic polymer, and the flowchart is given by Fig. 1 in terms of the molecular structures of the peptide at the final state of each simulation. As indicated in Fig. 1, 4 ns unconstrained simulation was first computed on the peptide with a starting structure of a linear chain (state A in Fig. 1). It was shown in Ref. [21] that the duration of $4 \mathrm{~ns}$ was enough for the peptide to relax into a conformational equilibrium. From the relaxed state (B in Fig. 1) a constrained simulation was then carried out to simulate the pulling process, with the position of the Leu1 residue being fixed and the Gly15 residue being pulled along the $x$-axis using a force of a $1000 \mathrm{~kJ} / \mathrm{mol} / \mathrm{nm}^{2}$ force constant at a pulling rate of $0.005 \mathrm{~nm} / \mathrm{ps}$. From the stretched structure of the peptide after 400 ps of the pulling simulation (state $\mathrm{C}$ in Fig. 1) three simulations were further calculated respectively: (1) $4 \mathrm{~ns}$ unconstrained simulation to capture the release process whose final structure is shown as D in Fig. 1, (2) $4 \mathrm{~ns}$ constrained simulation for holding the stretched peptide by reducing the pulling rate to zero (final state is indicated in E), and (3) constrained simulation that continued pulling the peptide with the same pulling rate of $0.005 \mathrm{~nm} / \mathrm{ps}$ for another $400 \mathrm{ps}$ to a more stretched state $(\mathrm{F}$ in Fig. 1). From state $\mathrm{F}$ finally, one more constrained simulation was performed to hold the peptide in this more stretched state for $4 \mathrm{~ns}$ and the ending structure is shown as G in Fig. 1.

\section{RESULTS AND DISCUSSION}

To verify the validity of the simulations, the radius of gyration $(R \mathrm{~g})$ of the $\alpha$ carbons $\left(C_{\alpha}\right)$ of the peptide was computed as a function of time for each simulation, and the results are plotted in Fig. 2. It is clear from Fig. 2 a that the $R \mathrm{~g}$ appears to become stable at a small value of approximately $0.6 \mathrm{~nm}$ after $1 \mathrm{~ns}$, indicating the peptide reaches a configurational equilibrium. Fig. $2 \mathrm{~b}$ shows an increasing trend for the $R \mathrm{~g}$ with time, highlighting the peptide is being stretched in the pulling simulation. From Fig. $2 \mathrm{c}$ it is observed that once the force is released, after $3 \mathrm{~nm}$ the $R \mathrm{~g}$ of the stretched peptide decreases to a small value that is similar to the relaxed one (i.e., $\sim 0.6 \mathrm{~nm}$ ), suggesting a similarity in geometric size between the relaxed peptide and the released peptide after being stretched. Fig. $2 \mathrm{c}$ also shows that in both simulations of hold 1 and hold 2 , the $R \mathrm{~g}$ remains stable after $2 \mathrm{~nm}$ at a higher value than $0.6 \mathrm{~nm}$, indicating the stretched states are held, respectively. The results of $R \mathrm{~g}$ plotted in Fig. 2, concomitant with the structures shown in Fig. 1, clearly demonstrate the deformations that the peptide undergoes in different simulations.

To further probe the deformations, the physical properties of the peptide were computed from the simulations of the peptide being in the relaxed state ( $B$ in Fig. 1), the released state (D in Fig. 1) and the stretched states ( $E$ and $G$ in Fig. 1), respectively, by averaging the values over the last nanosecond of each simulation, that is, from $3 \mathrm{~ns}$ to $4 \mathrm{~ns}$. The time-averaged results are tabulated in Table 1.

Table 1. Results averaged from $3 \mathrm{~ns}$ to $4 \mathrm{~ns}$ of simulations for the peptide [LGGVG] 3

\begin{tabular}{|c|c|c|c|c|}
\hline & relax & release & hold 1 & hold 2 \\
\hline$R \mathrm{~g}, \mathrm{~nm}^{\mathrm{a}}$ & $0.61 \pm 0.03$ & $0.61 \pm 0.02$ & $0.75 \pm 0.03$ & $1.26 \pm 0.04$ \\
\hline SASA, $\mathrm{nm}^{2 \mathrm{~b}}$ & $12.9 \pm 0.7$ & $12.6 \pm 0.6$ & $13.9 \pm 0.4$ & $16.4 \pm 0.4$ \\
\hline Number of side-chain contacts ${ }^{\mathrm{c}}$ & $50 \pm 5$ & $53 \pm 8$ & $49 \pm 5$ & $39 \pm 4$ \\
\hline Number of water molecules ${ }^{\mathrm{d}}$ & $112 \pm 7$ & $112 \pm 7$ & $116 \pm 6$ & $126 \pm 5$ \\
\hline Number of $\mathrm{H}$ bonds within peptide ${ }^{\mathrm{e}}$ & $2.32 \pm 1.15$ & $2.09 \pm 1.29$ & $1.91 \pm 0.65$ & $0.01 \pm 0.12$ \\
\hline Number of $\mathrm{H}$ bonds between peptide and water ${ }^{\mathrm{e}}$ & $26.9 \pm 3.1$ & $27.8 \pm 3.9$ & $29.6 \pm 2.7$ & $33.9 \pm 3.3$ \\
\hline$\tau_{c}$ of $\mathrm{H}$ bonds between peptide and water, $\mathrm{ps}^{\mathrm{f}}$ & 2.25 & 2.31 & 2.07 & 1.72 \\
\hline Vibrational energy, $10^{5} \mathrm{~kJ} / \mathrm{mol}^{\mathrm{g}}$ & 1.19 & 1.06 & 1.81 & 2.19 \\
\hline \multicolumn{5}{|c|}{$\begin{array}{l}\text { a Radius of gyration of the } \mathrm{C}_{\alpha .} \\
{ }^{\mathrm{b}} \text { Solvent accessible surface area was computed with the distance set to } 0.14 \mathrm{~nm} \text {. } \\
{ }^{\mathrm{c}} \text { Side-chain contacts were counted when two aliphatic carbon atoms were within } 0.54 \mathrm{~nm} \text { of each other, while atoms in neighboring } \\
\text { residues were not considered. } \\
{ }^{\mathrm{d}} \text { Number of water molecules were counted when a water molecule was within } 0.3 \mathrm{~nm} \text { of the peptide. } \\
{ }^{\mathrm{e}} \text { Hydrogen bonds were counted when the hydrogen and the acceptor were within } 0.35 \mathrm{~nm} \text { and the hydrogen bonding angle was within } 30^{\circ} \text {. } \\
{ }^{\mathrm{f}} \text { The life time }\left(\tau_{c}\right) \text { of the hydrogen bonds between peptide and water was determined by fitting to the autocorrelation function of the hydrogen bonds. } \\
{ }^{\mathrm{g}} \text { Vibrational energy of the peptide was determined from the quasi-harmonic frequencies. }\end{array}$} \\
\hline
\end{tabular}



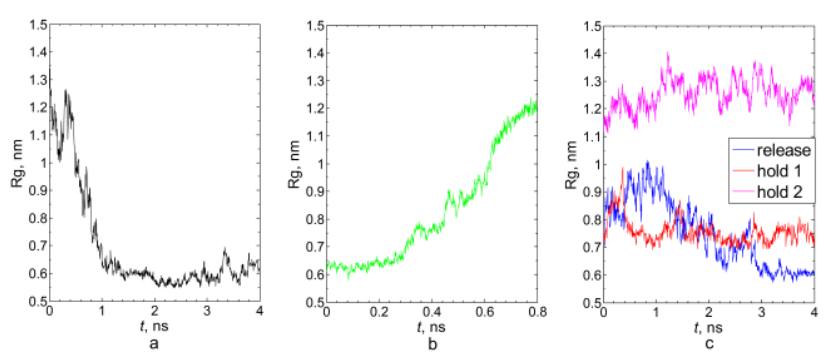

Fig. 2. Radius of gyration, $R g$, of $C_{\alpha}$ of the peptide [LGGVG] 3 as a function of time $t: \mathrm{a}$-the relax simulation corresponds to the process from $\mathrm{A}$ to $\mathrm{B}$ in Fig. $1 ; \mathrm{b}$-the pulling simulation resembles the evolution from $\mathrm{B}$ to $\mathrm{C}$ to $\mathrm{F}$ in Fig. 1; $\mathrm{c}-$ the simulations labeled 'release', 'hold 1 ' and 'hold 2' relate to the procedures from $\mathrm{C}$ to $\mathrm{D}, \mathrm{C}$ to $\mathrm{E}$, and $\mathrm{F}$ to $\mathrm{G}$ in Fig. 1, respectively

Table 1 presents the properties of $R \mathrm{~g}$ of the $C_{\alpha}$, solvent accessible surface area (SASA) of the peptide, number of side chain contacts, number of water molecules within $0.3 \mathrm{~nm}$ of the peptide, number of hydrogen bonds $(\mathrm{H}$ bonds) formed within the peptide, number and life time $\left(\tau_{c}\right)$ of hydrogen bonds between the peptide and water molecules, as well as vibrational energy of the peptide. Note that the metrics implemented to evaluate the physical properties are similar to that was employed in literatures $[20,21]$, and the details are given in the footnotes of the table.

Comparing the values of $R \mathrm{~g}$ in Table 1 , it is obvious that the geometric size of the released peptide is identical to that of the relaxed one, and that the sizes of the stretched ones are larger than the former two, with the peptide of hold 2 being the largest. This is a manifestation of the observations in Fig. 2. It is well known in the elastin community that elastin mimetic peptides tend to form a folded configuration when relaxed, resulting in a small radius of gyration. The larger values of $R \mathrm{~g}$ in hold 1 and hold 2 therefore indicate that an unfolded configuration is obtained for the peptide upon stress, which can also be visualized in Fig. 1.

Apart from $R \mathrm{~g}$, the values of all other properties tabulated in Table 1 are observed to be very close within error bars between the relaxed and the released states. This is a strong evidence that once the force is removed the stretched peptide returns to a state which is very similar to the relaxed state, that is to say, the deformation that the elastin mimetic peptide undergoes upon stress is elastic.

Referring to Table 1, it is also found that from the relaxed or released state to the states of hold 1 and hold 2 , there is an increasing trend in SASA, number of water molecules and number of $\mathrm{H}$ bonds between peptide and water, concomitant with a decreasing trend in number of side-chain contacts and number of $\mathrm{H}$ bonds within peptide. The above observations clearly illustrate that once the peptide is stressed an unfolded structure is formed, with the peptide being more exposed to the surrounding water molecules while the intramolecular contacts within the peptide being reduced.

With a careful check on the numbers of $\mathrm{H}$ bonds shown in Table 1 , it is arguable that the time-averaged values alone may not be sufficient to distinguish the states against each other. It is therefore more appropriate to study the histogram of the $\mathrm{H}$ bonds during the last nanosecond of each simulation, as presented in Fig. 3. It is obvious from Fig. 3 that for the $\mathrm{H}$ bonds both within the peptide and between the peptide and water, the histograms of the relaxed state and the released one are analogous, and are both different from the stretched states. As the stress is increased, the peak center of the profile for the $\mathrm{H}$ bonds within the peptide shifts to a lower value, while the one between peptide and water moves towards a larger value, resulting in the trend observed in the time-averaged $\mathrm{H}$ bonds values given in Table 1 .
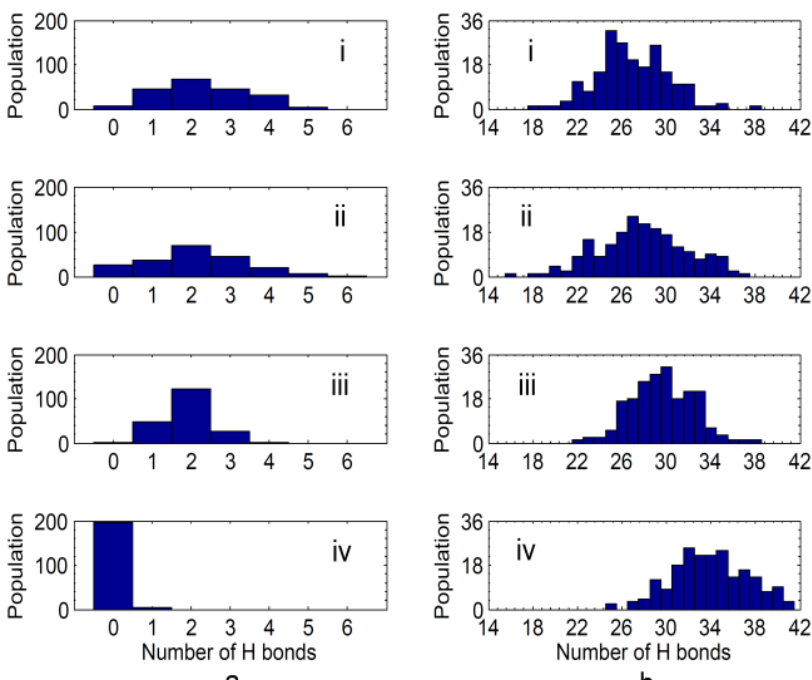

b

Fig. 3. Histograms of the $H$ bonds: $a$-within the peptide; $\mathrm{b}$-between the peptide and water. For both, plot i corresponds to the relaxed state in Table 1, ii the release, iii the hold 1 , and iv the hold 2 . The histograms were counted from 3 ns to 4 ns of each simulation

From the hydrogen bonds at any given time $t$, the autocorrelation function of all hydrogen bonds can be obtained by:

$$
\mathrm{C}(\tau)=<s_{i}(t) s_{i}(t+\tau)>,
$$

where $s_{i}(t)=[0,1]$ for $\mathrm{H}$ bond $i$ at time $t$. By fitting the autocorrelation function with multi-exponential fits detailed in Ref. [27], the lifetime, $\tau_{c}$, of the $\mathrm{H}$ bonds between peptide and water was determined, and the results are also shown in Table 1. It is clear that $\tau_{c}$ for hold 1 and hold 2 is shorter than that for relax or release, with hold 2 the shortest, indicating an increase in the mobility of the peptide-water system as the strain of the peptide is increased. A similar trend was also observed in our previous experimental study of localized water and protein backbone dynamics in mechanically strained elastin, where the correlation time of the random tumbling motion of water and the correlation times of the carbonyl and aliphatic carbons were all found to decrease, revealing also an increase in the mobilities of the localized water and the protein backbone upon strain [28]. This observation may be correlated to the increasing trend in the vibrational energy of the peptide presented also in Table 1, which reveals an increase in the peptide's mobility with increasing the strain. Note that the vibrational energy was computed from the quasi-harmonic frequencies in the 
quasi-harmonic approximation with the standard equation [29]:

$$
\mathrm{E}=\sum_{j}\left[\frac{\hbar \omega_{j}}{2}+\frac{\hbar \omega_{j}}{e^{\hbar \omega_{j} / k_{B} T}-1}\right],
$$

where $\hbar$ is the reduced Planck's constant; $k_{B}$ is the Boltzmann's constant; and $T$ is the temperature. $\omega_{j}$ is the quasi-harmonic frequency that was determined by the eigenvalues of the mass-weighted covariance matrix derived from the simulated trajectories [29]. Referring to Table 1 again, both the values of $\tau_{c}$ and vibrational energy are observed similar between the relaxed and the released peptide, indicating an elastic recovery of the system in terms of dynamics after the force is released.

Lastly, the Ramachandran plots of the leucine residues were computed to further probe the configurational properties of the peptide, and the results are given in Fig.4. It is evident that the Ramachandran plots for relax and release are similar, and are both different from the stretched ones. The major difference between the stretched ones and the relax or release one is that there is no population in the area around $(-600,-450)$ for hold 1 and hold 2, whereas some occurrences are present in this $\alpha$ helix region for the relax and the release, indicating that upon strain less configurations can be possessed by the peptide.
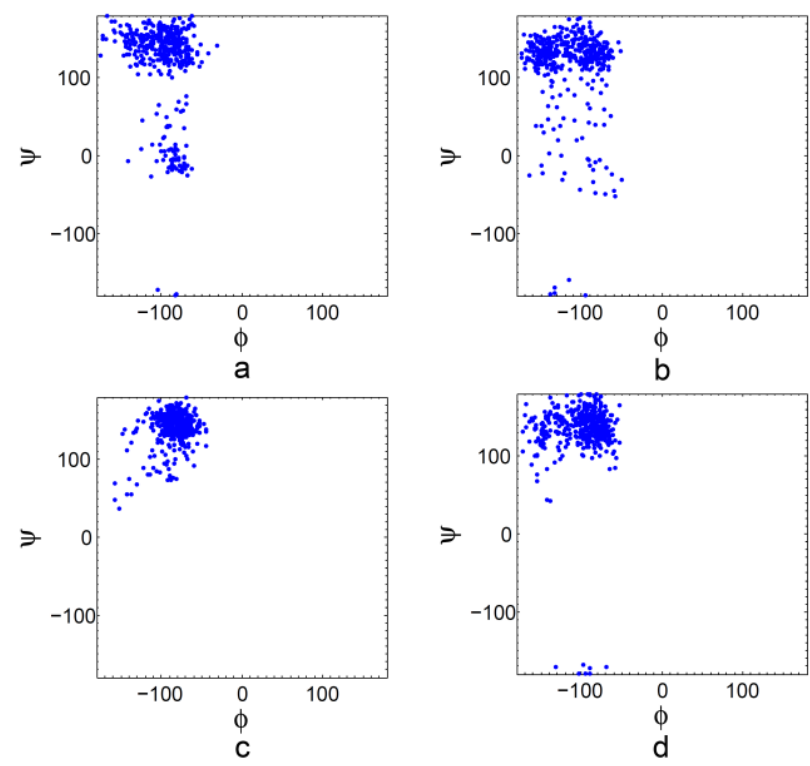

Fig. 4. Leucine Ramachandran plots computed from 3 ns to 4 ns of simulations for the peptide $\left[\mathrm{LGGVG}_{3}\right.$ in the state: $\mathrm{a}$-relax; b-release; c-hold $1 ; \mathrm{d}$-hold 2

\section{CONCLUSIONS}

To sum up, molecular dynamics simulations have been performed on the elastin mimetic peptide $[\mathrm{LGGVG}]_{3}$ to study the deformation of the peptide upon stress. The simulation results have demonstrated that once the peptide is stretched with stress, the configurational and dynamical properties are varied from the relaxed state, and that as the stress is increased, the variation is increased. The results have also shown that once the force is released, the configurational and dynamical properties return to a state which is almost identical to the relaxed one, revealing an elastic deformation of the elastin mimetic peptide. Based on this work, the simulation scheme used here may also be useful in studies of other elastic polymers.

\section{Acknowledgments}

C. Sun acknowledges support by Grant Number 31400718 from the National Natural Science Foundation of China.

\section{REFERENCES}

1. Hoeve, C.A.J., Flory, P.J. The Elastic Properties of Elastin Journal of the American Chemical Society 80 1958: pp. 6523-6526. https://doi.org/10.1021/ja01557a016

2. Gray, W.R., Sandberg, L.B., Foster, J.A. Molecular Model for Elastin Structure and Function Nature 246 1973: pp. $461-466$

3. Dorrington, K., Grut, W., McCrum, N.G. Mechanical State of Elastin Nature 255 1975: pp. 476-478.

4. Weis-Fogh, T., Andersen, S.O. New Molecular Model for the Long-Range Elasticity of Elastin Nature 227 1970: pp. $718-721$.

5. Urry, D.W., Parker, T.M. Mechanics of Elastin: Molecular Mechanism of Biological Elasticity and Its Relationship to Contraction Journal of Muscle Research and Cell Motility 23 2002: pp. 543-559. https://doi.org/10.1007/978-94-010-0147-2_14

6. Amruthwar, S.S., Janorkar, A.V. Preparation and Characterization of Elastin-Like Polypeptide Scaffolds for Local Delivery of Antibiotics and Proteins Journal of Materials Science: Materials in Medicine 23 2012: pp. 2903-2912.

https://doi.org/10.1007/s10856-012-4749-5

7. Kojima, C., Irie, K. Synthesis of Temperature-Dependent Elastin-Like Peptide-Modified Dendrimer for Drug Delivery Peptide Science 100 (6) 2013: pp. 714-721. https://doi.org/10.1002/bip.22276.

8. Sarangthem, V., Cho, E.A., Bae, S.M., Singh, T.D., Kim, S., Kim, S., Jeon, W.B., Lee, B., Park, R. Construction and Application of Elastin Like Polypeptide Containing IL-4 Receptor Targeting Peptide PLoS ONE 8 2013: pp. e81891. https://doi.org/10.1371/journal.pone.0081891

9. Amruthwar, S.S., $\quad$ Puckett, A.D., Janorkar, A.V. Preparation and Characterization of Novel Elastin-Like Polypeptide-Collagen Composites Journal of Biomedical Materials Research Part A 101A 2013: pp. 2383-2391. https://doi.org/10.1002/jbm.a.34514

10. Srokowski, E.M., Woodhouse, K.A. Surface and Adsorption Characteristics of Three Elastin-Like Polypeptide Coatings with Varying Sequence Lengths Journal of Materials Science: Materials in Medicine 24 2013: pp. $71-84$. https://doi.org/10.1007/s10856-012-4772-6

11. McDaniel, J.R., Radford, D.C., Chilkoti, A. A Unified Model for De Novo Design of Elastin-Like Polypeptides with Tunable Inverse Transition Temperatures Biomacromolecules 14 2013: pp. 2866-2872. https://doi.org/10.1021/bm4007166. Epub 2013 Jul 11.

12. Christensen, T., Hassouneh, W., Trabbic-Carlson, K., Chilkoti, A. Predicting Transition Temperatures of Elastin- 
Like Polypeptide Fusion Proteins Biomacromolecules 2013: pp. $1514-1519$.

https://doi.org/10.1021/bm400167h

13. Muiznieks, L.D., Weiss, A.S., Keeley, F.W. Structural Disorder and Dynamics of Elastin Biochemistry \& Cell Biology 88 2010: pp. 239-250.

https://doi.org/10.1139/o09-161

14. Reiersen, H., Clarke, A.R., Rees, A.R. Short Elastin-Like Peptides Exhibit the Same Temperature-Induced Structural Transitions as Elastin Polymers: Implications for Protein Engineering Journal of Molecular Biology 283 1998: pp. $255-264$. https://doi.org/10.1006/jmbi.1998.2067

15. Ma, X., Sun, C., Huang, J.X., Boutis, G.S. Thermal Hysteresis in the Backbone and Side-Chain Dynamics of the Elastin Mimetic Peptide [VPGVG] 3 Revealed by ${ }^{2} \mathrm{H}$ NMR Journal of Physical Chemistry B 116 2012: pp. 555-564. https://doi.org/10.1021/jp208966k

16. Martino, M., Coviello, A., Tamburro, A.M. Synthesis and Structural Characterization of Poly(LGGVG), an ElastinLike Polypeptide International Journal of Biological Macromolecules 27 2000: pp. 59-64.

https://doi.org/10.1016/S0141-8130(99)00118-X

17. Kumashiro, K.K., Kurano, T.L., Niemczura, W.P., Martino, M., Tamburro, A.M. ${ }^{13}$ C CPMAS NMR Studies of the Elastin-Like Polypeptide (LGGVG) $\mathrm{n}$ Biopolymers 70 2003: pp. $221-226$. https://doi.org/10.1002/bip.10470

18. Kumashiro, K.K., Ohgo, K., Niemczura, W.P., Onizuka, A.K., Asakura, T. Structural Insights into the Elastin Mimetic (LGGVG)6 Using Solid-State ${ }^{13} \mathrm{C}$ NMR Experiments and Statistical Analysis of the PDB Biopolymers 89 2008: pp. 668-679. https://doi.org/10.1002/bip.20984

19. Li, B., Alonso, D.O.V., Bennion, B.J., Daggett, V. Hydrophobic Hydration is an Important Source of Elasticity in Elastin-Based Biopolymers Journal of the American Chemical Society 123 2001: pp. 11991-11998. https://doi.org/10.1021/ja010363e

20. Li, B., Alonso, D.O.V., Daggett, V. The Molecular Basis for the Inverse Temperature Transition of Elastin Journal of Molecular Biology 305 2001: pp. 581-592. https://doi.org/10.1006/jmbi.2000.4306
21. Huang, J.X., Sun, C., Mitchell, O., Ng, N., Wang, Z.N., Boutis, G.S. On the Inverse Temperature Transition and Development of an Entropic Elastomeric Force of the Elastin Mimetic Peptide [LGGVG] 3,7 Journal of Chemical Physics 136 2012: pp. 085101. https://doi.org/10.1063/1.3685454

22. Hess, B., Kutnzer, C., Spoel, D.V.D., Lindahl, E. GROMACS 4: Algorithms for Highly Efficient, LoadBalanced, and Scalable Molecular Simulation Journal of Chemical Theory and Computation 4 2008: pp. 435-447. https://doi.org/10.1021/ct700301q

23. Berendsen, H.J.C., Postma, J.P.M., DiNola, A., Haak, J.R. Molecular Dynamics with Coupling to an External Bath Journal of Chemical Physics 81 1984: pp. $3684-3690$. https://doi.org/10.1063/1.448118

24. Jorgensen, W.L., Tirado-Rives, J. The OPLS Force Field for Proteins. Energy Minimizations for Crystals of Cyclic Peptides and Crambin Journal of the American Chemical Society 110 1988: pp. 1657-1666. https://doi.org/10.1021/ja00214a001

25. Teleman, O., Jonsson, B., Engstrom, S. A Molecular Dynamics Simulation of a Water Model with Intramolecular Degrees of Freedom Molecular Physics 60 1987: pp. $193-203$. https://doi.org/10.1080/00268978700100141

26. Zimmerman, K.J. Oral: All Purpose Molecular Mechanics Simulator and Energy Minimizer Journal of Computational Chemistry 12 1991: pp. 310-319. https://doi.org/10.1002/jcc.540120305

27. Spoel, D.V.D., Maaren, V., Larsson, P.J., Timneanu, N.P. Thermodynamics of Hydrogen Bonding in Hydrophilic and Hydrophobic Media Journal of Physical Chemistry B 110 2006: pp. 4393-4398. https://doi.org/10.1021/jp0572535

28. Sun, C., Mitchell, O., Huang, J.X., Boutis, G.S. NMR Studies of Localized Water and Protein Backbone Dynamics in Mechanically Strained Elastin Journal of Physical Chemistry B 115 2011: pp. 13935-13942. https://doi.org/10.1021/jp207607r

29. Andricioaei, I., Karplus, M. On the Calculation of Entropy from Covariance Matrices of the Atomic Fluctuations Journal of Chemical Physics 115 2001: pp. 6289-6292. https://doi.org/10.1063/1.1401821 